\title{
Interference Minimization in Hybrid WiFi/Cellular Networks
}

\author{
Petros S. Bithas*, Athanasios S. Lioumpas ${ }^{\natural}$, George K. Karagiannidis ${ }^{\dagger \ddagger}$ and Bayan S. Sharif ${ }^{\ddagger}$ \\ * Institute for Astronomy, Astrophysics, Space Applications and Remote Sensing, National Observatory of Athens \\ ${ }^{b}$ Cyta Hellas, Greece \\ ${ }^{\dagger}$ Department of Electrical and Computer Engineering Aristotle University of Thessaloniki, Thessaloniki, Greece \\ ${ }_{\ddagger}^{\ddagger}$ Electrical \& Computer Engineering Department, Khalifa University, UAE \\ Email:\{pbithas@iee.org, athanasios.lioumpas@hq.cyta.gr, geokarag@auth.gr\}
}

\begin{abstract}
The exploitation of already deployed wireless local area networks (WLAN)s access points (AP)s has attracted considerable attention, as an efficient and practical method to improve the performance of wireless networks. In this paper, we adopt a hybrid accessing approach, where the WLAN APs share their wireless cellular broadband connection with other users. These users will select their serving node based on a certain selection criterion. Thus a challenging research field is originated, where interfering effects and wireless resources limitations play a dominant role. Important performance metrics of the proposed hybrid scheme, including the bit error probability and the ergodic capacity, are theoretically studied and closed form expressions are derived. Numerical results and simulations show that the proposed wireless architecture may offer significant performance gains in the presence of multiple interferers, compared to a conventional cellular network.

Index Terms-Capacity, hybrid Cellular/WLAN, signal to interference and noise ratio, tethering.
\end{abstract}

\section{INTRODUCTION}

The demand for wireless data traffic is growing faster than the capacity provided by the network, due to the highly increased number of different type of connected devices. In addition, the link efficiency has reached its performance limits, while the increase of spectrum seems to be impractical. Thus, it is widely accepted that further spectral efficiency improvement is only possible by increasing the node deployment density [1]. Towards denser network deployments, two major approaches exist: a) network splitting into smaller macro-cells, and b) adoption of Heterogeneous Networks (HetNet)s concept. Cell splitting may not always be an efficient solution, especially in already dense deployments, where the additional intercell interference is prohibitive [1]. The concept of HetNets, relies on the deployment of heterogeneous low power nodes (LPN)s within the macro-cell [2], [3]. The HetNet deployments provide a wide area coverage through the macro cell and a more targeted coverage of special zones through the LPNs [4], [5]. The HetNet concept is also part of the 3rd Generation Partnership Project (3GPP) Long Term Evolution (LTE) network architecture, where the LPNs include femtocells, home eNodeBs (eNB)s and relay nodes [6].

The capacity offloading to non-cellular radio technologies, especially to 802.11-based wireless local area networks (WLAN)s (i.e., WiFi), has attracted considerable research interest as a costefficient, easy to deploy solution [7]. Taking into account the huge number of WiFi access points (AP)s already installed in several places, it becomes evident that a very dense network is already deployed. It is interesting to note that the IEEE 802.11 standard includes a convergence with 3GPP standards through the extensible authentication protocol-subscriber identity module protocol for authentication and key agreement protocol [8], which is an enabler for utilizing the WLAN APs for offloading cellular data in practice. In addition to offloading schemes, where the WLAN APs employ a wired backhaul, e.g., asymmetric digital subscriber line (ADSL), in [9] the authors presented an offloading scheme based on WiFi tethering, where the cellular radio links of one or more mobile smartphones are exploited for building WiFi hotspot, which can be then used by vicinal mobile users. Similar network setups have been also studied under the framework of cooperative relay-assisted network, e.g., [10].

In this paper, we adopt a hybrid cellular/WLAN communication approach, where the mobile users can be served by either the eNB or a WLAN AP, depending on the selection strategy (e.g., the signal to interference plus noise ratio (SINR)). In contrast to the conventional offloading approach, where the WLAN APs have a wired backhaul, a tethering approach is adopted. According to this scheme, the WLAN APs are wirelessly connected to the eNB and share this broadband connection with specific users over WLAN frequencies. Then the users select their serving node, i.e., the macro-cell eNB or a WLAN AP, based on a performance criterion. The aim of this architecture is to reduce the transmission power from the eNB to users with bad channel conditions (e.g., cell edge users) and thus the interference at both the cellular and WLAN part of the hybrid network, while avoiding modifications to the existing cellular network. Various performance metrics of the proposed scheme, namely the average bit error probability (ABEP), ergodic capacity and the outage probability (OP), are theoretically studied. For the reader's convenience, most of the notations and symbols used next are included in Table I.

\section{SYSTEM AND INTERFERENCE MODEL}

We consider the downlink of a wireless communication network, where the macro-cells are underlaid with $M$ wireless APs, which are not connected to a wired backhaul. Due to the frequency reuse employed in the macrocells, the user equipments (UE)s and the APs in each macrocell are subject to co-channel interference due to a number of nodes. The APs as well as the UEs are equipped with two radio access interfaces (RAI)s, namely a cellular RAI (e.g., LTE) and a WLAN RAI (e.g., WiFi), each operating at a different frequency band. Depending on the 
TABLE I

NOTATION AND SYMBOLS

\begin{tabular}{|c|c|}
\hline$z_{e u_{j, i}}$ & Complex channel gain between the $j$ th eNB and the $i$ th $\mathrm{UE}$ \\
\hline$z_{e a_{j, i}}$ & Complex channel gain between the $j$ th eNB and the $i$ th AP \\
\hline$z_{a u_{i, i}}$ & Complex channel gain between the $j$ th AP and the $i$ th UE \\
\hline$s_{e u_{j, i}}$ & Complex symbol transmitted by the $j$ th eNB targeting $i$ th $\mathrm{UE}$ \\
\hline$s_{e a}, i$ & Complex symbol transmitted by the $j$ th eNB targeting $i$ th AP \\
\hline$s_{a u_{j, i}}$ & Complex symbol transmitted by the $j$ th AP targeting $i$ th UE \\
\hline $\mathcal{I}_{U E, i}^{C}$ & Set of (cellular) interfering UEs to the $i$ th UE or AP \\
\hline $\mathcal{I}_{A P, i}^{C}$ & Set of (cellular) interfering APs to the $i$ th UE or AP \\
\hline $\mathcal{I}_{U E, i}^{W}$ & Set of (WLAN) interfering UEs to the $i$ th UE \\
\hline$z_{I_{e u, j}}$ & Complex channel gain of the $j$ th $\mathrm{UE} \in \mathcal{I}_{U E, i}^{C}$ \\
\hline$z_{I_{\mathfrak{a} u, j}}$ & Complex channel gain of the $j$ th AP $\in \mathcal{I}_{A P, i}^{C}$ \\
\hline$z_{I_{e, j}, j}$ & Complex channel gain of the $j$ th $\mathrm{UE} \in \mathcal{I}_{U E, i}^{W}$ \\
\hline$s_{I_{e u, j}}$ & Complex symbol transmitted by an eNB to the $j$ th $\mathrm{UE} \in \mathcal{I}_{U E, i}^{C}$ \\
\hline$s_{I_{e, j}, j}$ & Complex symbol transmitted by an eNB to the $j$ th $\mathrm{AP} \in \mathcal{I}_{A P, i}^{C}$ \\
\hline$s_{I_{\boldsymbol{a}, j, j}}$ & Complex symbol transmitted by an AP to the $j$ th $\mathrm{UE} \in \mathcal{I}_{U E, i}^{W}$ \\
\hline$P_{e u j, i}$ & $\begin{array}{l}\text { The power of the complex symbol transmitted by the } j \text { th eNB, } \\
\text { targeting the } i \text { th UE, } P_{e u_{j, i}}=\mathbb{E}\left\langle\left|s_{e u_{j, i}}\right|^{2}\right\rangle\end{array}$ \\
\hline$P_{e a_{j, i}}$ & $\begin{array}{l}\text { The power of the complex symbol transmitted by the } j \text { th eNB, } \\
\text { targeting the } i \text { th AP, } P_{e a_{j, i}}=\mathbb{E}\left\langle\left|s_{e a_{j, i}}\right|^{2}\right\rangle\end{array}$ \\
\hline$P_{a u_{j, i}}$ & $\begin{array}{l}\text { The power of the complex symbol transmitted by the } j \text { th AP, } \\
\text { targeting the } i \text { th UE, } P_{a u_{j, i}}=\mathbb{E}\left\langle\left|s_{a u_{j, i}}\right|^{2}\right\rangle\end{array}$ \\
\hline$P_{I_{e u, j}}$ & $\begin{array}{l}\text { The power of the complex symbol transmitted by an eNB, } \\
\text { targeting the } j \text { th } \mathrm{UE} \in \mathcal{I}_{U E, i}^{C}, P_{I_{e u, j}}=\mathbb{E}\left\langle\left|s_{I_{e u, j}}\right|^{2}\right\rangle\end{array}$ \\
\hline$P_{I_{e a, j}}$ & $\begin{array}{l}\text { The power of the complex symbol transmitted by the eNB, } \\
\text { targeting the } j \text { th } \mathrm{AP} \in \mathcal{I}_{A P, i}^{C}, P_{I_{e}, j}=\mathbb{E}\left\langle\left|s_{I_{e}, j}\right|^{2}\right\rangle\end{array}$ \\
\hline$P_{I_{a u, j}}$ & $\begin{array}{l}\text { The power of the complex symbol transmitted by an AP, } \\
\text { targeting the } j \text { th } \mathrm{UE} \in \mathcal{I}_{U E, i}^{W}, P_{I_{\boldsymbol{u}, j}}=\mathbb{E}\left\langle\left|s_{I_{\boldsymbol{u}, j}}\right|^{2}\right\rangle\end{array}$ \\
\hline
\end{tabular}

communication strategy (e.g., based on the received SINR), each UE might be served either through:

1) A direct communication link to the eNB (one phase communication, $\mathrm{eNB} \rightarrow \mathrm{UE}$ ) utilizing cellular frequencies.

2) An indirect link to the eNB via an AP, which forwards the received signal to the target UE (two-phase communication, $\mathrm{eNB} \rightarrow \mathrm{AP} \rightarrow \mathrm{UE}$ ), utilizing cellular frequencies for the $\mathrm{eNB} \rightarrow \mathrm{AP}$ link and WLAN frequencies for the $\mathrm{AP} \rightarrow \mathrm{UEs}$ link.

A UE, that is directly connected to the eNB, will experience interference caused by adjacent eNBs, which serve other UEs and APs at the same cellular frequencies. On the other hand, considering the eNB $\rightarrow \mathrm{AP} \rightarrow \mathrm{UE}$ scenario, in the first phase, the AP will experience interference from adjacent eNBs serving other UEs and APs at the same cellular frequencies. In the second phase the UE experiences interference from other APs that serve UEs at the same WLAN frequencies. Under these assumptions, the complex baseband signals transmitted by the $n$th eNB and received by the $k$ th UE can be expressed as

$$
\begin{aligned}
y_{e u_{n, k}}= & z_{e u_{n, k}} s_{e u_{n, k}}+\sum_{i=1}^{\left|\mathcal{I}_{U E, k}^{C}\right|} z_{I_{e u, i}} s_{I_{e u, i}} \\
& +\sum_{i=1}^{\left|\mathcal{I}_{A P, k}^{C}\right|} z_{I_{e a, i}} s_{I_{e a, i}}+w_{e u, k}
\end{aligned}
$$

with $|\mathcal{A}|$ denoting the size of a set $\mathcal{A}$. Moreover, the complex baseband signal transmitted by the $m$ th AP and received by the $k$ th UE is

$$
y_{a u_{m, k}}=z_{a u_{m, k}} s_{a u_{m, k}}+\sum_{i=1}^{\left|\mathcal{I}_{U E, k}^{W}\right|} z_{I_{a u, i}} s_{I_{a u, i}}+w_{a u, k}
$$

and that transmitted by the $n$th eNB and received by the $k$ th AP

$$
\begin{aligned}
& y_{e a_{n, k}}=z_{e a_{n, k}} s_{e a_{n, k}}+\sum_{i=1}^{\left|\mathcal{I}_{U E, k}^{C}\right|} z_{I_{e u, i}} s_{I_{e u, i}} \\
& +\sum_{i=1}^{\left|\mathcal{I}_{A P, k}^{C}\right|} z_{I_{e \bullet, i}} s_{I_{e \bullet, i}}+w_{e a, k} .
\end{aligned}
$$

In these equations $w_{e u, k}, w_{a u, k}$ and $w_{e a, k}$ denote the complex additive white Gaussian noise (AWGN) with zero mean and variance $N_{0}$ at the corresponding UEs and APs. Next we assume that the envelopes of channel complex gains, i.e., $h_{e u_{n, k}}, h_{e a_{n, k}}$, $h_{a u_{m, k}}, h_{I_{e u, i}}, h_{I_{\bullet u, i}}, h_{I_{e \bullet}, i}$, follow the Rayleigh distribution and hence their squares are exponentially distributed.

\section{A. Interference in a Conventional Cellular Network}

Considering a conventional cellular network, where the frequency resources are reused among macrocells. The instantaneous SINR of the $k$ th UE served by the $n$th eNB is

$$
\gamma_{c, k}=\frac{\gamma_{e u_{n, k}}}{1+\underbrace{\sum_{i=1, i \neq k}^{\left|\mathcal{I}_{U E, k}\right|} \gamma_{I_{e u, i}}}_{\text {eNB } \rightarrow \mathrm{UE}}}
$$

where $\gamma_{e u_{n, k}}=h_{e u_{n, k}}^{2} P_{e u_{n, k}} / N_{0}$ is the instantaneous received signal-to-noise ratio (SNR) of the $k$ th $\mathrm{UE}$ and $\gamma_{I_{e u, i}}=$ $h_{I_{e u, i}}^{2} P_{I_{e u, i}} / N_{0}$ is the interference-to-noise ratio (INR) due to the cellular co-channel interferers. Note that $\gamma_{e u_{n, k}}$ and $\gamma_{I_{e u, i}}$ are exponentially distributed random variables $(\mathrm{RV}) \mathrm{s}$ with mean $\bar{\gamma}_{e u_{n, k}}=\mathbb{E}\left\langle h_{e u_{n, k}}^{2}\right\rangle \frac{P_{e u_{n, k}}}{N_{\bullet}}$ and $\bar{\gamma}_{I_{e u, i}}=\mathbb{E}\left\langle h_{I_{e u, i}}^{2}\right\rangle \frac{P_{I_{e u, i}}}{N_{\bullet}}$, respectively, with $\mathbb{E}\langle\cdot\rangle$ denoting the statistical averaging.

\section{B. Interference in the Hybrid Cellular/WLAN Network}

Considering a hybrid cellular/WLAN network, where the UE is directly connected with the $n$th eNB (employing the cellular RAI), the instantaneous SINR of the $k$ th UE is

$$
\gamma_{e u, k}=\frac{\gamma_{e u_{n, k}}}{1+\underbrace{\sum_{i=1}^{\left|\mathcal{I}_{A P, k}^{C}\right|} \gamma_{I_{e \bullet, i}}}_{\mathrm{eNB} \rightarrow \mathrm{AP}}+\underbrace{\sum_{i=1}^{\left|\mathcal{I}_{U E, k}^{C}\right|} \gamma_{I_{e u, i}}}_{\mathrm{eNB} \rightarrow \mathrm{UE}}}
$$

where $\gamma_{I_{e_{e}, i}}=h_{I_{e \bullet}, i}^{2} P_{I_{e \bullet, i}} / N_{0}$ is the INR with mean $\bar{\gamma}_{I_{e \bullet}, i}=$ $\mathbb{E}\left\langle h_{I_{e}, i}^{2}\right\rangle P_{I_{e}, i} / N_{0}$. Considering a UE connected to a eNB via a WLAN AP. In the first transmission phase, the AP will experience interference from the eNB serving other UEs and APs at the same cellular frequencies. In the second phase, UE experiences interference from the APs that serve UEs at the same WLAN frequencies. In this sense, during the first phase, the instantaneous SINR at the $m$ th AP, will be

$$
\gamma_{e a, m}=\frac{\gamma_{e a_{n, m}}}{1+\underbrace{\sum_{i=1}^{\left|\mathcal{I}_{A \boldsymbol{P}, m}^{C}\right|} \gamma_{I_{e \bullet}, i}}_{\mathrm{e} N B \rightarrow A P}+\underbrace{\sum_{i=1}^{\left|\mathcal{I}_{U E, m}^{C}\right|} \gamma_{I_{e u, i}}}_{\mathrm{e} N B \rightarrow U E}}
$$

where $\gamma_{e a_{n, m}}=h_{e a_{n, m}}^{2} P_{e a_{n, m}} / N_{0}$ is the instantaneous SNR at the $m$ th AP with mean $\bar{\gamma}_{e a_{n, m}}=\mathbb{E}\left\langle h_{e a_{n, m}}^{2}\right\rangle \frac{P_{e \bullet n, m}}{N_{\bullet}}$. In the second phase, where the $k$ th UE receives the desired signal from one AP and interfering signals from the $m$ th APs, its instantaneous SINR is

$$
\gamma_{a u, k}=\frac{\gamma_{a u_{m, k}}}{1+\underbrace{\sum_{i=1}^{\left|\mathcal{I}_{U E, k}^{W}\right|} \gamma_{I_{\boldsymbol{\bullet}, i, i}}}_{\mathrm{A} P \rightarrow U E}}
$$


where $\left|\mathcal{I}_{U E, m}^{C}\right|+\left|\mathcal{I}_{U E, k}^{W}\right|=N, \gamma_{a u_{m, k}}=h_{a u_{m, k}}^{2} P_{a u_{m, k}} / N_{0}$ is the instantaneous SNR at the $k$ th UE and $\gamma_{I_{a, i}}=$ $h_{I_{a u, i}}^{2} P_{I_{\mathbf{a}^{u}, i}} / N_{0}$ is the INR due to WLAN co-channel interferers, with mean values $\bar{\gamma}_{a u_{m, k}}=\mathbb{E}\left\langle h_{a u_{m, k}}^{2}\right\rangle P_{a u_{m, k}} / N_{0}$ and $\bar{\gamma}_{I_{\boldsymbol{a} u, i}}=\mathbb{E}\left\langle h_{I_{\boldsymbol{\bullet} u, i}}^{2}\right\rangle P_{I_{\boldsymbol{\bullet} u, i}} / N_{0}$, respectively. Note that the sets $\mathcal{I}_{U E, i}^{C}$ and $\mathcal{I}_{A P, i}^{C}$ do not include the same UEs and APs when considering the direct or indirect communication scenarios.

The communication mode of each UE (i.e., direct or via an WLAN AP) is determined by the eNB, which has a full knowledge of the all links conditions within the cell. In this work, we assume that this decision is based on the SINR, namely the UE connects to that node (eNB or WLAN AP) that results in the maximum individual instantaneous SINR.

\section{SINR ANALYSIS}

Here, the probability density function (PDF) and cumulative distribution function (CDF) of the output SINR, are presented for both networks under consideration. Due to space limitations, details on the derivation procedures were omitted.

\section{A. Statistical Analysis}

1) SINR Statistics for the Conventional Cellular Network: Let us consider the conventional cellular network and the SINR at the $k$ th UE as given by (4). In this study, it is assumed that the co-channel interfering signals add up incoherently since this represents a more realistic assessment of the co-channel interference in cellular systems [11]. The total instantaneous INR caused by other eNBs to the $k$ th UE, is given by

$$
\gamma_{c_{I, k}}=\sum_{i=1}^{\left|\mathcal{I}_{U E, k}^{C}\right|} \gamma_{I_{e u, i}} \text {. }
$$

For independent and identical distributed (i.i.d.) fading, the PDF and CDF of $\gamma_{c, k}$ are as follows

$$
\begin{aligned}
& f_{C}(\gamma)=\frac{Z^{-X}}{Y} \frac{\exp \left(-\frac{\gamma}{Y}\right)}{\left(\frac{\gamma}{Y}+\frac{1}{Z}\right)^{X}}\left(1+\frac{X}{\frac{\gamma}{Y}+\frac{1}{Z}}\right) \\
& F_{C}(\gamma)=1-\frac{1}{Z^{X}}\left(\frac{\gamma}{Y}+\frac{1}{Z}\right)^{-X} \exp \left(-\frac{\gamma}{Y}\right)
\end{aligned}
$$

where $C=\gamma_{c, k}, X=\left|\mathcal{I}_{U E, k}^{C}\right|, Y=\bar{\gamma}_{e u_{n, k}}$ and $Z=\bar{\gamma}_{I_{e u, k}}$.

2) SINR Statistics for the Hybrid Cellular/WLAN Network:

For the hybrid cellular/WLAN network, the following two complementary communication cases are investigated, namely onephase direct $\mathrm{eNB} \rightarrow \mathrm{UE}$ communication and two-phase indirect $\mathrm{eNB} \rightarrow \mathrm{AP} \rightarrow \mathrm{UE}$ communication.

a) Direct Communication: In this case, the SINR at the target UE is given by (5). The PDFs of the total instantaneous INR caused to the $k$ th UE by the eNB serving UEs at the same frequency, i.e.,

$$
\gamma_{I_{e u, k}}=\sum_{i=1}^{\left|\mathcal{I}_{U E, k}^{C}\right|} \gamma_{I_{e u, i}}
$$

and that of the total instantaneous INR caused to the $k$ th UE by the eNB serving APs at the same frequency, i.e.,

$$
\gamma_{I_{e \bullet, k}}=\sum_{i=1}^{\left|\mathcal{I}_{A P, k}^{C}\right|} \gamma_{I_{e \bullet, i}}
$$

follow the chi-square distribution. For i.i.d. fading conditions, the PDF and the CDF of $\gamma_{c, k}$ is given in (12) (shown at the top of the next page). In (12), $C=\gamma_{e u, k}, X_{1}=\left|\mathcal{I}_{U E, k}^{C}\right|, X_{2}=\left|\mathcal{I}_{A P, k}^{C}\right|$, $Y=\bar{\gamma}_{e u_{n, k}}, Z_{1}=\bar{\gamma}_{I_{e u, k}}, Z_{2}=\bar{\gamma}_{I_{e \cdot k},}$. b) Indirect Communication: In the case of the indirect connection of the UE to the eNB, there are two communication phases. During the first one (i.e., eNB $\rightarrow$ AP), the SINR at the target AP is given by (6), while in the second phase one (i.e, $\mathrm{AP} \rightarrow \mathrm{UE})$, the instantaneous SINR at the target UE is expressed as in (7). Assuming i.i.d. fading conditions, for the first phase, the PDF and the CDF of $\gamma_{e a, m}$ is given in (12), by substituting $C=\gamma_{e a, m}, X_{1}=\left|\mathcal{I}_{U E, m}^{C}\right|, X_{2}=\left|\mathcal{I}_{A P, m}^{C}\right|, Y=\bar{\gamma}_{e a_{n, m}}$, $Z_{1}=\bar{\gamma}_{I_{e u, m}}, Z_{2}=\bar{\gamma}_{I_{e, m}, m}$. For the second phase, the PDF and the CDF of $\gamma_{a u, k}$ is given in (9), by substituting $C=\gamma_{a u, k}$, $X=\left|\mathcal{I}_{U E, k}^{W}\right|, Y=\bar{\gamma}_{a u_{m, k}}$ and $Z=\bar{\gamma}_{I_{a, u}, k}$.

Indirect Communications Total Instantaneous SINR: In case where the AP decodes and forwards the data to the target UE, the instantaneous SINR at the output of the eNB $\rightarrow$ APs $\rightarrow$ UEs link can be tightly approximated in the high SNR regime as [2] $\gamma_{e a u, k}(\gamma)=\min \left(\gamma_{e a, k}, \gamma_{a u, k}\right)$ and thus the corresponding CDF of $\gamma_{e a u, k}$ can be expressed as

$$
\begin{aligned}
& F_{\gamma_{e \bullet u, k}}(\gamma)=\operatorname{Pr}\left\{\min \left(\gamma_{e a, k}, \gamma_{a u, k}\right)<\gamma\right\} \\
& =1-\left[1-F_{\gamma_{e \bullet, k}}(\gamma)\right]\left[1-F_{\gamma_{\bullet u, k}}(\gamma)\right] \\
& =F_{\gamma_{e \bullet, k}}(\gamma)+F_{\gamma_{\bullet u, k}}(\gamma)-F_{\gamma_{e \bullet, k}}(\gamma) F_{\gamma_{e \bullet, k}}(\gamma)
\end{aligned}
$$

Furthermore, the corresponding PDF expression of $\gamma_{e a u, k}$ is the following

$$
\begin{aligned}
& f_{\gamma_{e \bullet u, k}}(\gamma)=f_{\gamma_{e, k}}(\gamma)+f_{\gamma_{\bullet, u}, k}(\gamma) \\
& -f_{\gamma_{e \bullet, k}}(\gamma) F_{\gamma_{\mathfrak{a} u, k}}(\gamma)-f_{\gamma_{\mathfrak{a} u, k}}(\gamma) F_{\gamma_{e \bullet, k}}(\gamma) \text {. }
\end{aligned}
$$

c) Total Hybrid Cellular/W N Network Output SINR: Since in the mode of operation, (eNB $\rightarrow \mathrm{UE}$ or $\mathrm{eNB} \rightarrow \mathrm{AP} \rightarrow \mathrm{UE}$ ), the UE selects the communication link to be connected that provides the maximum SINR, the total instantaneous endto-end SINR can be finally expressed as [12] $\gamma_{\text {tot, } \mathrm{k}}(\gamma)=$ $\max \left(\gamma_{e u, k}, \gamma_{e a u, k}\right)$. Therefore, the CDF of $\gamma_{\text {tot,k }}$ is given by

$$
F_{\gamma_{\text {tot, }}}(\gamma)=F_{\gamma_{e u, k}}(\gamma) F_{\gamma_{e a u, k}}(\gamma)
$$

while the corresponding PDF expression can be obtained as

$$
f_{\gamma_{\mathrm{tot}, \mathrm{k}}}(\gamma)=f_{\gamma_{e u, k}}(\gamma) F_{\gamma_{e a u, k}}(\gamma)+F_{\gamma_{e u, k}}(\gamma) f_{\gamma_{e a u, k}}(\gamma)
$$

\section{B. Performance Evaluation}

Here, using the previous derived expressions for the PDF and the CDF of the output SINR, the ABEP, ergodic capacity and OP criteria will be studied.

1) Average Bit Error Probability $(A B E P)$ : For several modulation schemes, the system's ABEP, $P_{b e}$, can be evaluated employing the PDF-based approach as follows

$$
P_{b e}=A \int_{0}^{\infty} \exp (-B \gamma) f_{\gamma_{\mathrm{t} \cdot \mathrm{t}, \mathrm{k}}}(\gamma) d \gamma
$$

where $(A, B)$ are constants that depend on the modulation type. Substituting in (17) the PDF expression provided in (9) (for the conventional cellular case) or (12) (for the hybrid cellular/WLAN case), integrals of the following form appear

$$
\begin{aligned}
\mathcal{T}_{1, i}(\underbrace{a_{i}, b_{i}, c_{i}}_{i \in\{1,3\}})=\int_{0}^{\infty} & \exp \left[-\left(B+\sum_{j=1}^{i} c_{j}\right) x\right] \\
& \times\left[\prod_{j=1}^{i}\left(\frac{1}{x+a_{j}}\right)^{b_{j}}\right] d x
\end{aligned}
$$




$$
\begin{aligned}
f_{C}(\gamma) & =\frac{\frac{1}{Y}}{Z_{2, k}^{X_{2}} \Gamma\left(X_{2}\right)} \frac{\exp (-\gamma / Y)}{Z_{1, k}^{X} \Gamma\left(X_{1}\right)} \sum_{j=0}^{X_{2}-1} \frac{\left(\begin{array}{c}
X_{2}-1 \\
j
\end{array}\right)(-1)^{j} \Gamma\left(X_{1}+j\right)}{\left(\frac{1}{Z_{1, k}}-\frac{1}{Z_{1, k}}\right)^{j+X_{1}}}\left\{\frac{\Gamma\left(X_{2}-j\right)}{\left(\frac{\gamma}{Y}+\frac{1}{Z_{1, k}}\right)^{X_{2}-j}}\right. \\
& \left.\times\left(1+\frac{X_{2}-j}{\frac{\gamma}{Y}+\frac{1}{Z_{1, k}}}\right)-\sum_{k=0}^{X_{1}+j-1} \frac{\Gamma\left(X_{2}+k-j\right)}{k !} \frac{\left(\frac{1}{Z_{1, k}}-\frac{1}{Z_{1, k}}\right)^{k}}{\left(\frac{\gamma}{Y}+\frac{1}{Z_{1, k}}\right)^{X_{2}+k-j}}\left(1+\frac{X_{2}+k-j}{\frac{\gamma}{Y}+\frac{1}{Z_{1, k}}}\right)\right\} \\
F_{C}(\gamma) & =\frac{1}{Z_{1, k}^{X_{2}} \Gamma\left(X_{2}\right)} \frac{1}{Z_{1, k}^{X_{1}} \Gamma\left(X_{1}\right)} \sum_{j=0}^{X_{2}-1} \frac{(-1)^{j}\left(\begin{array}{c}
X_{2}-1 \\
j
\end{array}\right) \Gamma\left(X_{1}+j\right)}{\left(1 / Z_{1, k}-1 / Z_{2, k}\right)^{X_{1}+j}}\left\{\left[\frac{\Gamma\left(X_{2}-j\right)}{Z_{2, k}^{j-X_{2}}}-\sum_{n=0}^{X_{1}+j-1} \frac{\left(1 / Z_{1, k}-1 / Z_{2, k}\right)^{n}}{n !} \frac{\Gamma\left(X_{2}+n-j\right)}{\left.Z_{1, k}^{j-X_{2}-n}\right]}\right.\right. \\
& \left.-\exp \left(-\frac{\gamma}{Y}\right)\left[\frac{\Gamma\left(X_{2}-j\right)}{\left(\gamma / Y+1 / Z_{2, k}\right)^{X_{2}-j}}-\sum_{n=0}^{X_{1}+j-1} \frac{\left(1 / Z_{1, k}-1 / Z_{1, k}\right)^{n}}{\left(\gamma / Z_{1, k}+1 / Z_{1, k}\right)^{X_{2}+n-j}} \frac{\Gamma\left(X_{2}-j+n\right)}{n !}\right]\right\}
\end{aligned}
$$

where $a_{i}, c_{i} \in \mathbb{R}, b_{i} \in \mathbb{N}$. By employing partial fraction and using [13, eq. (3.382/4)], the following closed-form expression for $\mathcal{T}_{1, i}\left(a_{i}, b_{i}, c_{i}\right)$ can be derived as

$$
\begin{aligned}
& \mathcal{T}_{1, i}\left(a_{i}, b_{i}, c_{i}\right)=\sum_{j=1}^{i} \sum_{h=1}^{b_{j}} \Xi_{j, h}\left(B+\sum_{j=1}^{i} c_{j}\right)^{h-1} \\
& \times \exp \left[a_{j}\left(B+\sum_{j=1}^{i} c_{j}\right)\right] \Gamma\left[1-h,\left(B+\sum_{j=1}^{i} c_{j}\right)\right]
\end{aligned}
$$

where $\Xi_{j, h}=\left.\frac{1}{\left(b_{j}-h\right) !} \frac{d^{\mathbf{b}_{j}-h}}{d \gamma^{\boldsymbol{b}_{j}-h}} \prod_{\substack{p=1 \\ \text { f }}}^{i}\left(x+a_{p}\right)^{-b_{\boldsymbol{p}}}\right|_{x=-a_{j}}$ and $\Gamma(\cdot, \cdot)$ represents the upper incomplete Gamma function [13, eq. (8.350/1)]. Based on (19), a simplified expression for the ABEP of the conventional cellular scenario is given by

$$
\begin{aligned}
& P_{b e}^{c}=\frac{A}{\bar{\gamma}_{e u_{n, k}}}\left(\frac{\bar{\gamma}_{e u_{n, k}}}{\bar{\gamma}_{I_{e u, k}}}\right)^{\left|\mathcal{I}_{U E, k}^{C}\right|}\left(\frac{1}{\bar{\gamma}_{e u_{n, k}}}+B\right)^{\left|\mathcal{I}_{U E, k}^{C}\right|-1} \\
& \times \exp \left[\frac{\bar{\gamma}_{e u_{n, k}}}{\bar{\gamma}_{I_{e u, k}}}\left(\frac{1}{\bar{\gamma}_{e u, k}}+B\right)\right] \\
& \times\left\{\Gamma\left[1-\left|\mathcal{I}_{U E, k}^{C}\right|, \frac{\bar{\gamma}_{e u_{n, k}}}{\bar{\gamma}_{I_{e u, k}}}\left(\frac{1}{\bar{\gamma}_{e u_{n, k}}}+B\right)\right]\right. \\
& \left.+\frac{\left(\frac{1}{\bar{\gamma}_{e u_{n, k}}}+B\right)}{\left(\left|\mathcal{I}_{U E, k}^{C}\right| \bar{\gamma}_{e u_{n, k}}\right)^{-1}} \Gamma\left[-\left|\mathcal{I}_{U E, k}^{C}\right|, \frac{\bar{\gamma}_{e u_{n, k}}}{\bar{\gamma}_{I_{e u, k}}}\left(\frac{1}{\bar{\gamma}_{e u_{n, k}}}+B\right)\right]\right\} .
\end{aligned}
$$

2) Ergodic Capacity: In our system, the overall achievable capacity is given by

$$
C_{\gamma_{\mathrm{tot}, \mathrm{k}}}=\frac{1}{N H} \int_{0}^{\infty} \log _{2}(1+\gamma) f_{\gamma_{\mathrm{tot}, \mathrm{k}}}(\gamma) d \gamma
$$

where $N H$ denotes the number of hops.

a) Exact Expression: Substituting in this definition the PDF expression provided in (9) or (12), and using the infinite series representation of the $\log _{2}(\cdot)$ function, i.e., [14, eq. (4.1.29)], integrals of the following form appear

$\mathcal{T}_{3, i}(\underbrace{a_{i}, b_{i}, c_{o}}_{i \in\{1,4\}})=\int_{0}^{\infty} x^{D} \exp \left(-\sum_{j=1}^{i} c_{j} x\right) \prod_{j=1}^{i+1}\left(\frac{1}{x+a_{j}}\right)^{b_{j}} d x$.
Employing the partial fraction and using [15, eq. (2.3.6/9)], $\mathcal{T}_{3, i}\left(a_{i}, b_{i}, c_{i}\right)$ can be solved in closed form as

$$
\begin{aligned}
\mathcal{T}_{3, i}\left(a_{i}, b_{i}, c_{i}\right)= & \sum_{j=1}^{i+1} \sum_{h=1}^{b_{j}} \Xi_{j, h} \Gamma(D+1) a_{j}^{D+1-h} \\
& \times \Psi\left[D+1, D+2-h,\left(\sum_{j=1}^{i} c_{j}\right)\right] .
\end{aligned}
$$

It is noted that the previously presented approach, for obtaining the ABEP and capacity, can be directly applied to the hybrid cellular/WLAN case. However, the derived expressions for these metrics are not included here due to space limitations.

3) Outage Probability: The OP is defined as the probability that the SINR falls below a predetermined threshold $\gamma_{\text {th }}$ and is given by $P_{\gamma_{\text {tot, }}}=F_{\gamma_{\text {tot, }}}\left(\gamma_{\mathrm{th}}\right)$. Therefore, the OP can be evaluated by using the CDF expression provided in (9) or (12).

\section{Numerical Results AND Discussion}

Here, we present and discuss several numerical results, which demonstrate the performance of the proposed architecture compared to the conventional cellular system, which serves as a benchmark. In Fig. 1, an OP comparison between the conventional scheme and the proposed hybrid approach is presented as a function of $\gamma_{\mathrm{th}}$. In this figure, for the conventional cellular scheme we have assumed $\left|\mathcal{I}_{U E, k}^{C}\right|=24$, for the hybrid $\left|\mathcal{I}_{U E, k}^{C}\right|=\left|\mathcal{I}_{A P, k}^{C}\right|=\left|\mathcal{I}_{U E}^{W}\right|=12$, while in all cases the average transmit power is equal to $40 \mathrm{~dB}$. It is depicted that the hybrid scheme provides a significant performance improvement. Additionally the performance gap between these two communication approaches increases as the average INR decreases. In Fig. 2, considering average transmit power equal to $40 \mathrm{~dB}$, the ABEP as well as channel capacity of both schemes are plotted as a function of the number of interferers also for various values of the average INR. In this figure, it is clearly depicted that the ABEP performance improves by employing the hybrid scheme. However, since the relaying scheme under consideration is one way, its information theoretic capacity is divided by a factor of two, resulting in an decrease in the overall hybrid scheme capacity. However, in many works it has been shown that by adopting a two-way relay channel, it is possible to remove the $1 / 2$ loss rate loss factor in the capacity, e.g., [16]. Following such an approach in our case, the hybrid system capacity is considerably improved, thus providing the best performance in 


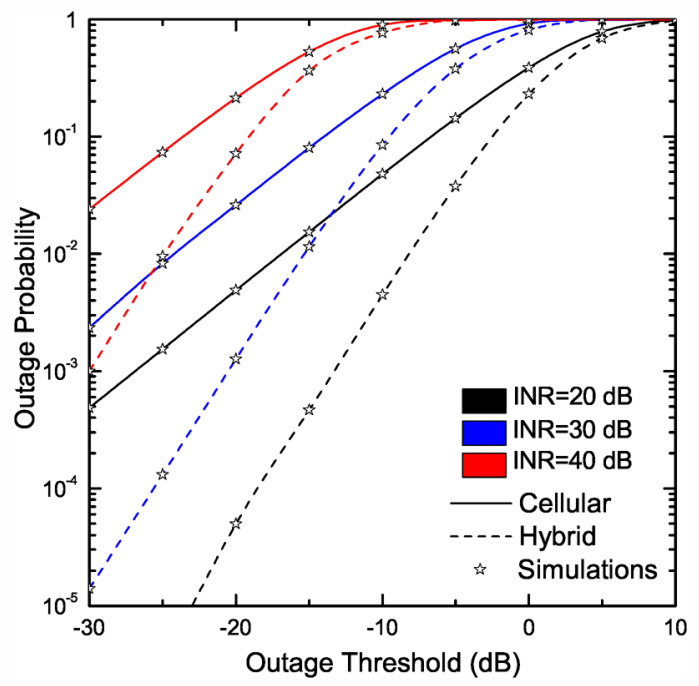

Fig. 1. OP vs outage threshold for various values of the average INR.
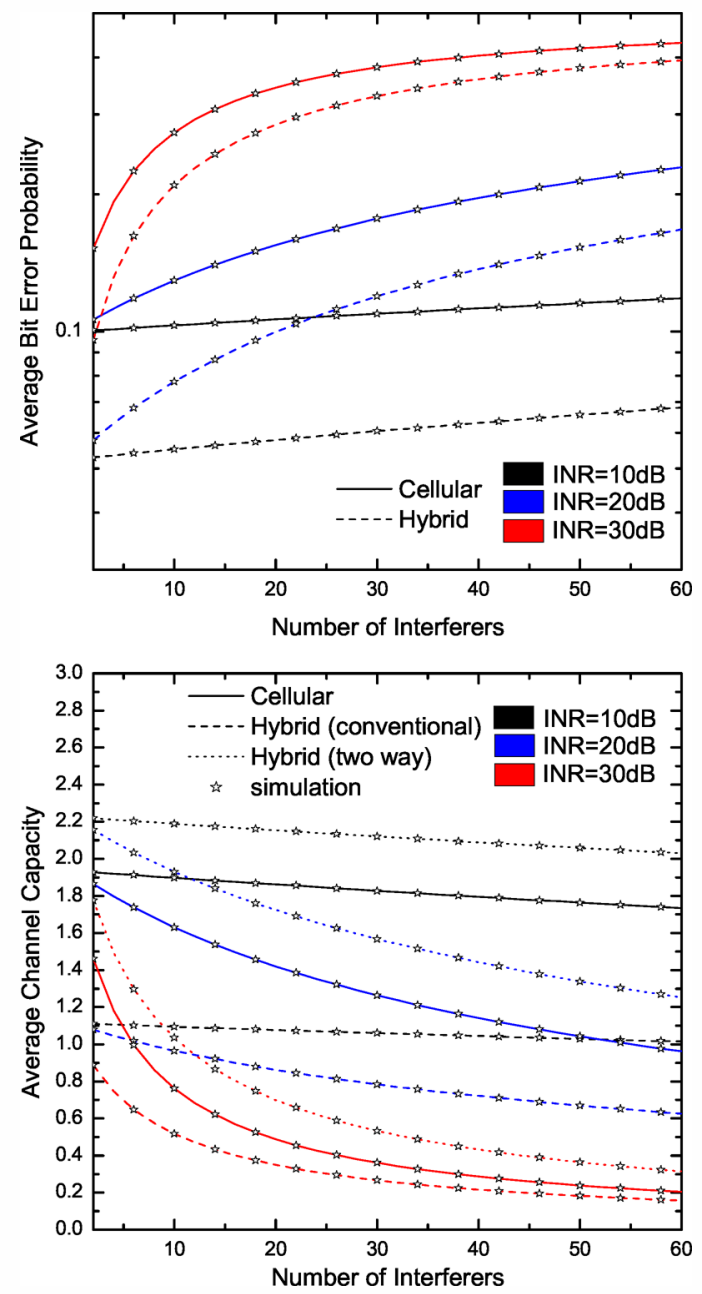

Fig. 2. The ABEP and channel capacity vs the number of interferers.

all cases. Finally, for comparison purposes, computer simulation results are also included in Figs. 1-2, verifying the validity of the proposed theoretical approach.

\section{CONCLUSIONS}

In this paper, we investigated the performance improvement induced by adopting a hybrid cellular/WLAN communication architecture where the mobile users can be served by either the eNB or a WLAN AP. In the proposed scheme the WLAN APs are wirelessly connected to the eNB and share this broadband connection with other users over WLAN frequencies. Important performance metrics of the proposed hybrid scheme including the ABEP, capacity and OP were theoretically studied. Numerical results showed that the proposed wireless architectures may offer significant performance gains in the presence of multiple interferers, compared to a conventional cellular network.

\section{ACKNOWLEDGEMENT}

The research leading to these results has received funding from the European Union's Seventh Framework Programme (FP7/2007-2013) under grant agreement $n^{\circ} 609094$.

\section{REFERENCES}

[1] A. Damnjanovic, J. Montojo, Y. Wei, T. Ji, T. Luo, M. Vajapeyam, T. Yoo, O. Song, and D. Malladi, "A survey on 3GPP heterogeneous networks," IEEE Wireless Commun., vol. 18, no. 3, pp. 10-21, Jun. 2011.

[2] T. Q. Duong, V. N. Q. Bao, and H.-J. Zepernick, "On the performance of selection decode-and-forward relay networks over Nakagami- $m$ fading channels," IEEE Comm. Lett., vol. 13, no. 3, pp. 172-174, Mar. 2009.

[3] H. A. Suraweera, D.S. Michalopoulos and C. Yuen "Performance analysis of fixed gain relay systems with a single interferer in Nakagami- $m$ fading channels," IEEE Trans. Veh. Technol., vol. 61, no. 3, pp. 1457-1463, Mar 2012.

[4] P. Li and Y. Fang "On the throughput capacity of heterogeneous wireless networks," IEEE Trans. Mobile Comput., vol. 11, no. 12, pp. 2073-2086, Dec. 2012

[5] M. Z. Shakir and M.-S. Alouini "On the area spectral efficiency improvement of heterogeneous network by exploiting the integration of macrofemto cellular networks," IEEE International Conference on Communications, pp. 5695-5700, Jun. 2012.

[6] 3GPP Evolved Universal Terrestrial Radio Access (EUTRA) and Evolved Universal Terrestrial Radio Access Network (E-UTRAN); Overall Description; Stage 2 (Release 10).

[7] F. Zhang, W. Zhang, and Q. Ling, "Noncooperative game for capacity offload," IEEE Trans. Wireless Commun., vol. 11, no. 4, pp. 1565-1575, Apr. 2012.

[8] IEEE, IEEE standard for local and metropolitan area networks - part 11: Wireless lans (2012).

[9] A. Sharma, V. Navda, R. Ramjee, V. N. Padmanabhan, and E. M. Belding, "Cool-Tether: Energy efficient on-the-fly WiFi hot-spots using mobile phones," in 5th International Conference on Emerging Networking Experiments and Technologies, Dec. 2009.

[10] M. Xia, and S. Aissa, "Cooperative AF relaying in spectrum-sharing systems: Outage probability analysis under co-channel interferences and relay selection," IEEE Trans. on Commun., vol. 60, no. 11, pp. 3252-3262, Nov. 2012.

[11] R. Prasad and A. Kegel, "Improved assessement of interference limits in cellular radio performance," IEEE Trans. Veh. Technol., vol. 40, pp. 412419, May. 1991.

[12] K. K. Jin, T. Q. Duong and H. V. Poor, "Outage Probability of SingleCarrier Cooperative Spectrum Sharing Systems with Decode-and-Forward Relaying and Selection Combining," IEEE Trans. Wirel. Commun., vol. 12, no. 2, pp. 806-817, Feb. 2013.

[13] I. S. Gradshteyn and I. M. Ryzhik "Table of Integrals, Series, and Products," Academic Press, 2000, New York

[14] M. Abramowitz and I. A. Stegun. "Handbook of Mathematical Functions with Formulas, Graphs, and Mathematical Tables," National Bureau of Standards Applied Mathematic, 1972, New York.

[15] A. P. Prudnikov and Y. A. Brychkov and O. I. Marichev "Integrals and Series, Volume 1," Gordon and Breach Science Publishers, 1986.

[16] B. Rankov and A. Wittneben, "Achievable rate regions for the two-way relay channel,"in IEEE Int. Symposium on Informat. Theory, Jul. 2006. 